\title{
Landauer and Thouless Conductance: a Band Random Matrix Approach
}

\author{
Giulio Casati $^{(a, b)}$, Italo Guarneri ${ }^{(a, c)}$, Giulio Maspero \\ International Center for the Study of Dynamical Systems, University of Milan at Como, \\ via Lucini 3, 22100 Como, Italy \\ (a) Istituto Nazionale di Fisica della Materia, Unitá di Milano, via Celoria 16, 20133, \\ Milan, Italy \\ (b) I.N.F.N., Sezione di Milano, via Celoria 16, 20133, Milan, Italy \\ (c) I.N.F.N., Sezione di Pavia, via Bassi 6, 27100, Pavia, Italy
}

PACS numbers: 05.45.+b, 72.15.Rn, 72.10.-d

Section: Condensed Matter

G.Maspero

e-mail: maspero@mvcomo.fis.unico.it

fax: ++3931326230 


\begin{abstract}
We numerically analyze the transmission through a thin disordered wire of finite length attached to perfect leads, by making use of banded random Hamiltonian matrices. We compare the Landauer and the Thouless conductances, and find that they are proportional to each other in the diffusive regime, while in the localized regime the Landauer conductance is approximately proportional to the square of the Thouless one. Fluctuations of the Landauer conductance were also numerically computed; they are shown to slowly approach the theoretically predicted value.
\end{abstract}


Two main theoretical approaches to the study of electronic conduction in disordered wires at zero temperature have been developed, starting from ideas originally due to Thouless and Landauer, respectively. In the Thouless approach, the wire is regarded as a closed system, and its conductance is defined through the sensitivity of eigenvalues to changes of the boundary conditions [1,2]. In the Landauer approach [3, 团 the wire is an open system, connected to perfect conductors, and its conductance is related to the transparency of the wire to electronic waves, via a formula, that can be derived from linear response theory [5]. In the metallic regime, the conductance is given by the Thouless formula, which can in turn be derived from the Kubo-Greenwood formula supplemented by certain randommatrix theoretical assumption [6]. The relation between the above two approaches is a subtle theoretical problem.

In this Letter we present a comparison of the Thouless and the Landauer conductance, on a model which allows for an efficient numerical computation of both. In this model, the wire is described by a 'Band Random Matrix' (BRM), that is, a real symmetric matrix $H$ of rank $L$ such that matrix elements $h_{i j} \neq 0$ only for $|i-j| \leq b+1$, where $b$ is the halfwidth of the band. We chose Gaussian distribution for all non-zero elements, with variance $\frac{\alpha^{2}}{2}$ for those on the side diagonals and $\alpha^{2}$ for those on the main diagonal . Strictly speaking, BRMs of this kind describe 1D disordered wires with long-range hopping; however, they are also deemed to catch most of the general features of quasi-one dimensional models [7].

In the Landauer approach the wire is connected on both sides to ideal conductors, or "leads". In our model the electron dynamics in the leads is described by two semi-infinite BRMs with the same bandwidth $b$, with all matrix elements inside the band equal to 1 . The Schrödinger equation is

$$
\sum_{k=-(b+1)}^{b+1} \mathcal{H}_{i, i+k} u_{i+k}=E u_{i}
$$

where $\mathcal{H}_{i, j}$ are the non-zero elements of the Hamiltonian $\mathcal{H}$, which is an infinite band matrix. Elements $\mathcal{H}_{i, j}$ are the same as above defined $h_{i, j}$ for $1 \leq i, j \leq L$ and are equal to 1 otherwise. In this way, one can write $\mathcal{H}=\mathcal{H}_{0}+V$, where $\mathcal{H}_{0}$ is an infinite band matrix, with constant 
unit elements inside the band, which describes free motion, and $V$ is a scattering potential. The original BRM $H$ is just the projection of the Hamiltonian $\mathcal{H}$ on the subspace spanned by states $|i\rangle, 1 \leq i \leq L$.

Free waves (which are eigenfunctions of $\mathcal{H}_{0}$ ) are of the form $u_{m}=\frac{1}{\sqrt{2 \pi}} e^{i k m}$, with the wave number $k$ obeying the dispersion law

$$
\frac{\sin \left(\frac{2 b+1}{2} k\right)}{\sin \left(\frac{k}{2}\right)}=E .
$$

The left hand side of (2) is a periodic function of $k$ of period $2 \pi$, which takes the value $2 b+1$ when its argument is a multiple of $2 \pi$. For $-1<E<+1$ Eq.(2) has $2 b$ solutions, which can be found analytically for $E=1, E=0$ and $E=-1$ or by numerical methods at any other value of $E$. For example, they are $k_{j}=\frac{2 \pi j}{2 b+1}$ with $j=1, \ldots, 2 b$ for $E=0$ and $k_{s}=\frac{2 \pi s}{b}$ with $s=1, \ldots, b-1$ and $k_{t}=\frac{2 \pi t+\pi}{b+1}$ with $t=0, \ldots, b$ for $E=1$. The various allowed values of the wave-number $k$ at a given energy $E$ correspond to different scattering channels and in the range $1 \geq E \geq-1$ all scattering channels are open. We thus have a multi-channel scattering problem with exactly $b$ ingoing and $b$ outgoing channels, which, if $1<<b<<L$, formally reproduces the case of a quasi-one dimensional wire.

The scattering properties of the conductor are described by a unitary scattering matrix $S$, that relates incoming and outgoing amplitudes, $I_{L(R)}$ and $O_{L(R)}$ :

$$
S\left(\begin{array}{c}
I_{L} \\
I_{R}
\end{array}\right)=\left(\begin{array}{c}
O_{L} \\
O_{R}
\end{array}\right)
$$

where the subscripts $L$ and $R$ stand for the left and the right lead. The $S$ matrix can be written as

$$
S=\left[\begin{array}{cc}
r & t \\
t^{\prime} & r^{\prime}
\end{array}\right]
$$

where $t$ and $t^{\prime}$ are the transmission sub-matrices in the two opposite directions, and $r$ and $r^{\prime}$ are the reflection sub-matrices.

The Landauer conductance is 8

$$
\mathcal{G}=\frac{e^{2}}{2 \pi \hbar} \sum_{i j}\left|t_{i j}\right|^{2},
$$


where $t_{i j}$ are the elements of the $t$ sub-matrix in (3). We have computed the S-matrix by standard methods, namely by numerically solving the Lippman-Schwinger equation

$$
u^{ \pm}-G_{0}^{ \pm} \cdot V u^{ \pm}=u
$$

where $u$ are free eigenfunctions, $u^{ \pm}$interacting ones. The free Green function, $G_{0}^{ \pm}=(E-$ $\left.H_{0} \pm i \epsilon\right)_{\epsilon=0}^{-1}$, has matrix elements

$$
\begin{aligned}
\left(G_{0}^{ \pm}\right)_{n, m} & =\left.\left\langle n\left|\left(E-H_{0} \pm i \epsilon\right)^{-1}\right| m\right\rangle\right|_{\epsilon=0} \\
& =\left.\frac{1}{2 \pi} \int_{0}^{2 \pi} d k \frac{e^{i(m-n) k}}{E \pm i \epsilon-\frac{\sin \left(\frac{2 b+1}{2} k\right)}{\sin \left(\frac{k}{2}\right)}}\right|_{\epsilon=0}
\end{aligned}
$$

which were numerically computed by exploiting the Residues Theorem. Finally, the scattering matrix was computed via the standard formula:

$$
S_{i j}=\delta_{i j}-\left.\left.2 \pi i \sqrt{\frac{d k_{i}}{d H}}\right|_{H=E} \sqrt{\frac{d k_{j}}{d H}}\right|_{H=E}<u_{i}|V| u_{j}^{ \pm}>
$$

The unitarity condition $S^{+} S=I$ provides a check on the precision of the results, and was fulfilled within a typical error $10^{-10}$.

We have considered the dimensionless residual conductance

$$
g=\frac{2 \pi \hbar}{e^{2}} \mathcal{G}
$$

and investigated the scaling properties of the geometric average $g_{a v}=\exp \overline{\langle\ln g\rangle}$ in the regime $1 \ll b \ll L$, where $L$ is the length of the disordered sample. The brackets $\langle\ldots\rangle$ mean here average over different realizations of the potential (20000 realizations in our computations) while the bar means average over the energy range $(-1,1)$. As expected, $g_{a v}$ turned out to depend on $b$ and $L$ only through the localization parameter $x=\frac{b^{2}}{L}$ (Fig.1), where the physical meaning of $b$ and $b^{2}$ is that they are proportional respectively to the mean free path and to the localization length. For $x \gg 1$, i.e. in the delocalized regime, this dependence has the form $g_{a v} \propto x \propto L^{-1}$, which is the "ohmic" behavior (Fig. 2). Instead, in the localized regime $x \ll 1, g_{a v} \approx \exp (-c / x)$ (Fig. 3).

Our results can be summarized by the following interpolating law, valid in all regimes: 


$$
g_{a v}=\frac{a+b \exp (-d / x)}{\exp (c / x)-1}
$$

where $c \approx 2.70$ and $d \approx 0.55$ (obtained from the fitting in the localized region) and $a \approx 6.58$ and $b \approx-5.53$ (obtained from the fitting in the metallic region). If the conductance is computed without performing the energy average, a similar scaling law is obtained, though with slightly different constants (curves $\mathbf{a}$ and $\mathbf{b}$ in Fig. 4).

We now turn to the Thouless approach. In order to define the (dimensionless) Thouless conductance, the BRM which describes the disordered sample has to be 'periodicized' as described in ref. [9]. The 'periodic' BRM thus obtained describes a ring-shaped conductor, and depends on a phase $\phi$ that has the meaning of an Aharonov-Bohm flux switched on through the ring. The disorder-averaged Thouless conductance is

$$
\mathcal{K}=\frac{1}{\Delta}\left\langle\left.\left|\frac{d^{2} E}{d \phi^{2}}\right|\right|_{\phi=0}\right\rangle
$$

where $\Delta$ is the mean level spacing, and $\langle\ldots\rangle$ denotes statistical averaging. Although the scaling behavior of $\mathcal{K}$ was described in ref. [9] by an interpolating law different from Eq. (8), the latter law yields a slightly better fit with the parameters $a^{\prime} \approx 0.11, b^{\prime} \approx-3.1 \cdot 10^{-2}$, $c^{\prime} \approx 1.56$ and $d^{\prime} \approx 0.99$. Therefore Eq. (8) can be used to describe the scaling of both the Landauer and the Thouless conductance, albeit with different parameters.

A key problem in comparing the Thouless and the Landauer conductance is connected with the matching of the "free" and of the "disordered" part of the Hamiltonian, i.e., with the effect of "contacts", which was discussed in refs. [10. If the arbitrary scale factor $\alpha$ appearing in the disordered part of our Hamiltonian $\mathcal{H}$ is varied, while keeping the free Hamiltonian $\mathcal{H}_{0}$ unchanged, the Thouless conductance is not affected, nor is the value of the localization length in the 'infinite' sample. The Landauer conductance, instead, is to some extent modified. It becomes very small both at small and at large values of $\alpha$, and it has a relatively broad maximum around $\alpha=1$. A value of $\alpha$ close to 1 is also found on requiring that the local density of states for the "free" dynamics be the same as the density of interacting states in the sample (having computed the latter from the interacting Green 
function, in a standard way). Our choice of $\alpha=1$, which maximizes the transmission, is very similar to the "matching wire" prescription, suggested by Economou and Soukoulis [11].

The comparison between Landauer and Thouless conductance, computed as described above, is shown in Fig.4. In particular, in the metallic regime $(x \gg 1)$ we have $g_{a v} \approx(7.5 \pm$ 0.4) $K_{a v}+0.8$, that is, the two conductances are proportional in the diffusive regime, where (9) is assumed to be valid. This difference cannot be removed by using of a different expression for the Landauer formula, as in $[8]$. The meaning of the coefficent of proportionality $\approx$ $7.5 \pm 0.4$ is not clear to us [12. It is of course possible that this coefficient becomes closer to 1 in the limit $b \rightarrow \infty, L \rightarrow \infty, b^{2} / L=$ const.; this we were unable to check due to obvious numerical limitations, but, in the parameter range we were able to explore, the variation of this factor is quite slight.

In the localized regime $(x \ll 1)$ we get $\ln \left(g_{a v}\right) \approx \beta \ln \left(K_{a v}\right)+5.66$, that is to say, the Landauer conductance is proportional to $K_{a v}^{\beta}$, with $\beta=1.7$. The error in this numerical estimate is of order 0.1 , due to the difficulty in computing curvatures in the localized regime. In this connection, we have also studied two different tridiagonal models: a BRM with $b=1$ and a standard Anderson model with Gaussian disorder on the main diagonal. Comparing Landauer conductances and curvatures on these two models we got $\beta_{B R M}=2.08 \pm 0.04$ and $\beta_{\text {And }}=2.09 \pm 0.01$ [13]. It seems therefore reasonable [14 to conjecture $\beta=2$.

Let us now discuss the fluctuation properties of the conductance. First, we have found that in the localized region the following relation holds: $\operatorname{Var}(\ln g)=-\kappa\langle\ln g\rangle+\gamma$ with $\kappa=2.00 \pm 0.02$, and $\gamma=-4.3 \pm 0.5$ (Fig.5). This has to be compared with the variance of the curvature, which in [9] was found to obey a similar law, but with $\kappa \approx 1$. This fact reflects the most serious difference between Landauer conductance and curvature in the strongly localized regime.

We have also performed a detailed analysis of the distribution $P(g)$ of conductance in different regimes. Our results are in perfect agreement with theoretical expectations for quasi-1D conductors [15], in both the localized and in the metallic regimes. In particular, 
we have found that the shape of this distribution is controlled by the same scaling parameter $x$ : it is log-normal in the localized region, $x \ll 1$, and normal in the delocalized one, $x \gg 1$.

Special attention was paid to universal conductance fluctuations in the metallic regime. We have performed numerical simulations for large values of the scaling parameter $\frac{b^{2}}{L}$, which is proportional to the ratio of the localization length (in the infinite sample) to the finite sample size. At the same time, we have taken the ratio $\frac{b}{L}$ small, that is, we have taken samples considerably larger than the mean free path. Our data for the variance of conductance versus the scaling parameter are summarized in Fig. 6 . For not very large values of $x \approx 5$ the value of $\operatorname{Var}(g)$ remains close to $\frac{1}{8}$, slightly different from the expected value $\frac{2}{15}$. As discussed in [16], the value $\frac{1}{8}$ is predicted by RMT, while $\frac{2}{15}$ follows from different theoretical approaches, based either on diagrammatic calculations, or on a diffusion-equation approach for transfer matrices; it is also obtained from RMT, if an appropriate repulsion law is used for eigenvalues of the transfer matrix. More detailed numerical studies, at larger values of $x \approx 10$, reveal that $\frac{2}{15}$ is slowly approached on increasing $x$. All our results were carefully checked by using a very large number of realizations, and by implementing different random number generators.

Our data indicate that the value $\frac{2}{15}$ is approached very slowly, in such a way that actual data linger around $\frac{1}{8}$ in a significant parameter range. That the approach to $\frac{2}{15}$ can be relatively slow has been pointed out in Ref. [10], where this effect was related to the coupling with channels.

Finally we would like to remark that, since the velocity in the $i$ th channel is $v_{i}=\left.\frac{d H}{d k_{i}}\right|_{H=E}$, in our model different channels have different velocities. This raises the question, how does this fact affect our results. We have then modified the structure of the leads, in such a way that the dispersion law gives the same velocity in all channels. For this we have chosen the free hamiltonian with elements $H_{i j}=\delta_{i j}+\delta_{i+b+1, j}+\delta_{i-b-1, j}$. The data obtained in this way are practically indistinguishable from those in Fig. 1. Therefore the fluctuation properties of conductance appear to be independent of the specific properties of the leads.

In conclusion, we have studied the Landauer conductance for thin disorderd wires at- 
tached to perfect leads in 1D (or in quasi-1D geometry). The model we have used is based on Band Random Matrices with the modifications imposed by the presence of the leads. The results are compared with those found for the Thouless conductance both in localized and metallic regimes. In the localized regime our results reveal that the Landauer conductance is proportional to the square of the Thouless conductance. In the metallic regime they are proportional to each other. Therefore, our data confirm theoretical expectations for the fluctuation properties of conductance in the metallic regime. 


\section{REFERENCES}

[1] D.Thouless J.T.Edwards. J.Phys., C5, (1972) 807.

[2] D.Thouless. Phys.Rep., 13, (1974) 93.

[3] R. Landauer. I. B. M. Res. Dev., 1, (1957).

[4] R. Landauer. Philos. Mag., 21, (1970) 873.

[5] D.S.Fisher and P.A.Lee. Phys. Rev., B23, (1981) 6851.

[6] E.Akkermans and G.Montambaux. Phys.Rev.Lett., 68, (1992) 642.

[7] Y.V.Fyodorov and A.D.Mirlin. Int.J.Mod.Phys., 8, (1994) 3795.

[8] M.Büttiker, Y.Imry, R.Landauer, and S.Pinhas. Phys. Rev., B31, (1985) 6207.

[9] G.Casati, I.Guarneri, F.M.Izrailev, L.Molinari, and K.Życzkowski. Phys.Rev.Lett., 72, (1994) 2697.

[10] S. Iida, H. A. Weidenmüller and J.A.Zuk. Ann. Phys. (NY), 200, (1990) 219.

[11] E.N.Economou and C.M.Soukoulis. Phys. Rev. Lett., 43, (1981) 618.

[12] After the conclusion of this work, we became aware of the related work:

D.Braun, E.Hofstetter, G.Montambaux and A.MacKinnon. preprint cond-mat/9611059

[13] These results were obtained computing curvatures by means of a formula valid only for tridiagonal models, which was pointed out to us by Luca Molinari.

[14] An argument to the effect that the dissipative conductance, in the deeply localized regime, should be proportional to the square of the cur' is based on the fact that in the Fourier expansion of energy levels with respect to the phase $\phi$ only the lowest harmonics survive. This argument was shown to us by Eric Akkermans.

[15] J.L.Pichard, N.Zanon, Y.Imry, and A.D.Stone. J.Phys.(France), 51, (1990) 587. 
[16] C.W.J. Beenakker. Mod. Phys. Lett., B8, (1994) 469. 


\section{FIGURES}

FIG. 1. Logarithm of the geometric average of the Landauer conductance $g_{a v}=\exp \overline{\langle\ln (g)\rangle}$ vs. logarithm of the scaling parameter $x=\frac{b^{2}}{L}$. Numerical data correspond to different values of $L$ and $b$ in the ranges $50 \leq L \leq 1500,7 \leq b \leq 80$. Triangles are for the dispersion law (2), circles for a dispersion law giving the same velocity in all channels. The full line gives the fitting law eq.(8).

FIG. 2. Geometric average of Landauer conductance vs. the scaling parameter $\frac{b^{2}}{L}$ in the delocalized regime $\left(b^{2} \gg L\right)$. The linear fit shows the Ohmic behavior of Landauer conductance: $g_{a v}=\exp \overline{\langle\ln (g)\rangle}=A \frac{b^{2}}{L}+B$, with $A=0.44 \pm 10^{-2}, B=0.6 \pm 0.1$.

FIG. 3. $\ln \left(g_{a v}\right)$ vs. the inverse of the scaling parameter in the localized regime $\left(b^{2} \ll L\right)$. The linear fit shows the exponential decrease of Landauer conductance due to localization: $\overline{\langle\ln (g)\rangle}=-C \frac{L}{b^{2}}+D$, with $C=2.70 \pm 4 \cdot 10^{-2}, D=1.8 \pm 0.2$.

FIG. 4. Comparison between the fittings of Landauer conductance, computed with (curve $a$ ) and without (curve $b$ ) an energy average in the interval $(-1,1)$. Notice that the effect of the energy average is very small. Curve c gives the fit of Thouless conductance computed in ref.[8].

FIG. 5. Variance of the logarithm of Landauer conductance $g$ vs. $<\ln (g)>$. The numerical fit shows that in the localized regime $\operatorname{Var}(\ln (g))=-\kappa\langle\ln (g)\rangle+\gamma$, with $\kappa=2.00 \pm 0.02$ and $\gamma=-4.3 \pm 0.5$

FIG. 6. Variance of the logarithm of Landauer conductance vs. logarithm of the scaling parameter: the plateau in which $\operatorname{Var}(g)$ is independent of $b$ corresponds to the regime of UCF, before the onset of the ballistic regime. The theoretical expected value $\frac{2}{15}$ is reached only for $x \approx 10$, while for $x \approx 5$ data linger around $\frac{1}{8}$ (upper and lower horizontal lines). 


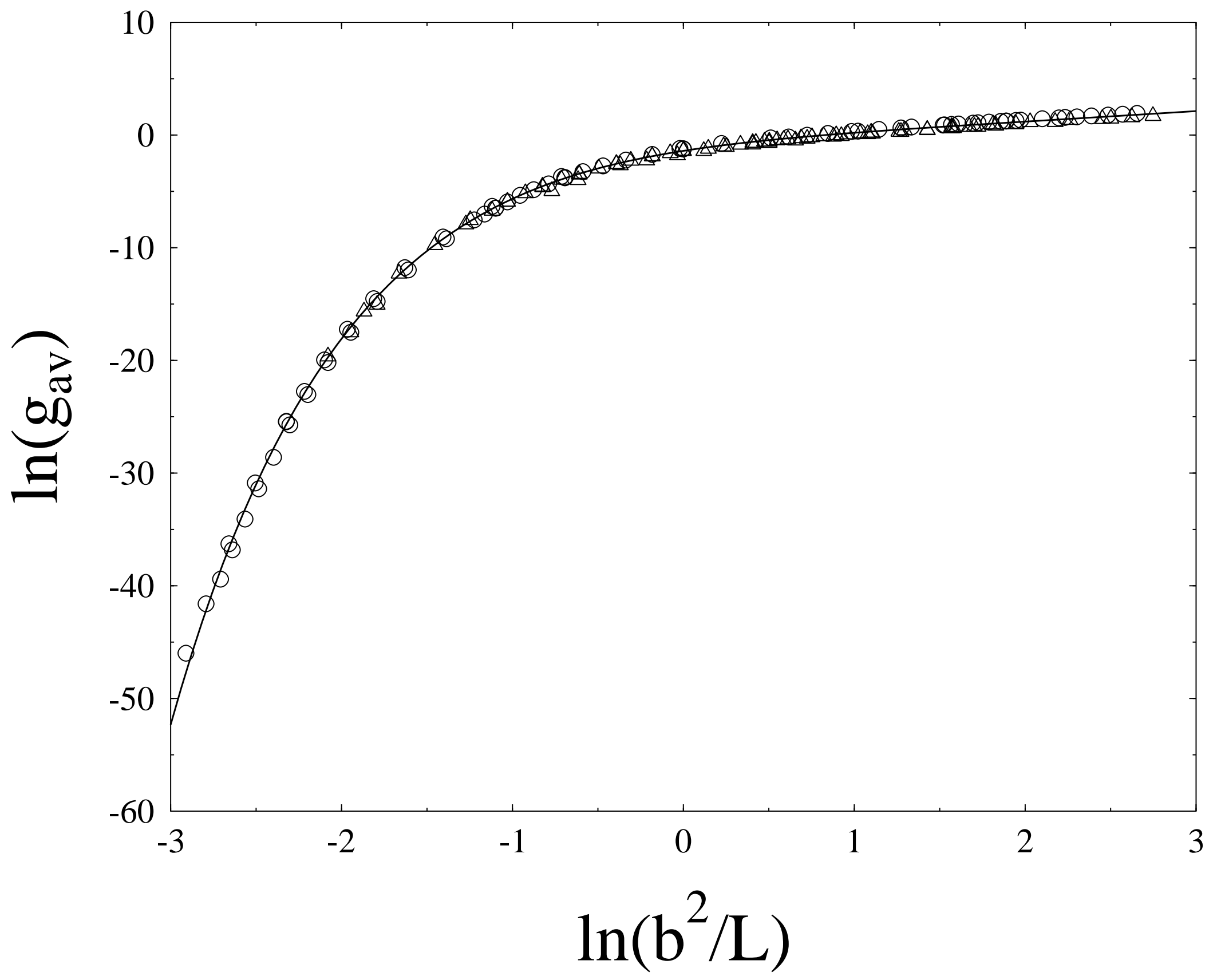




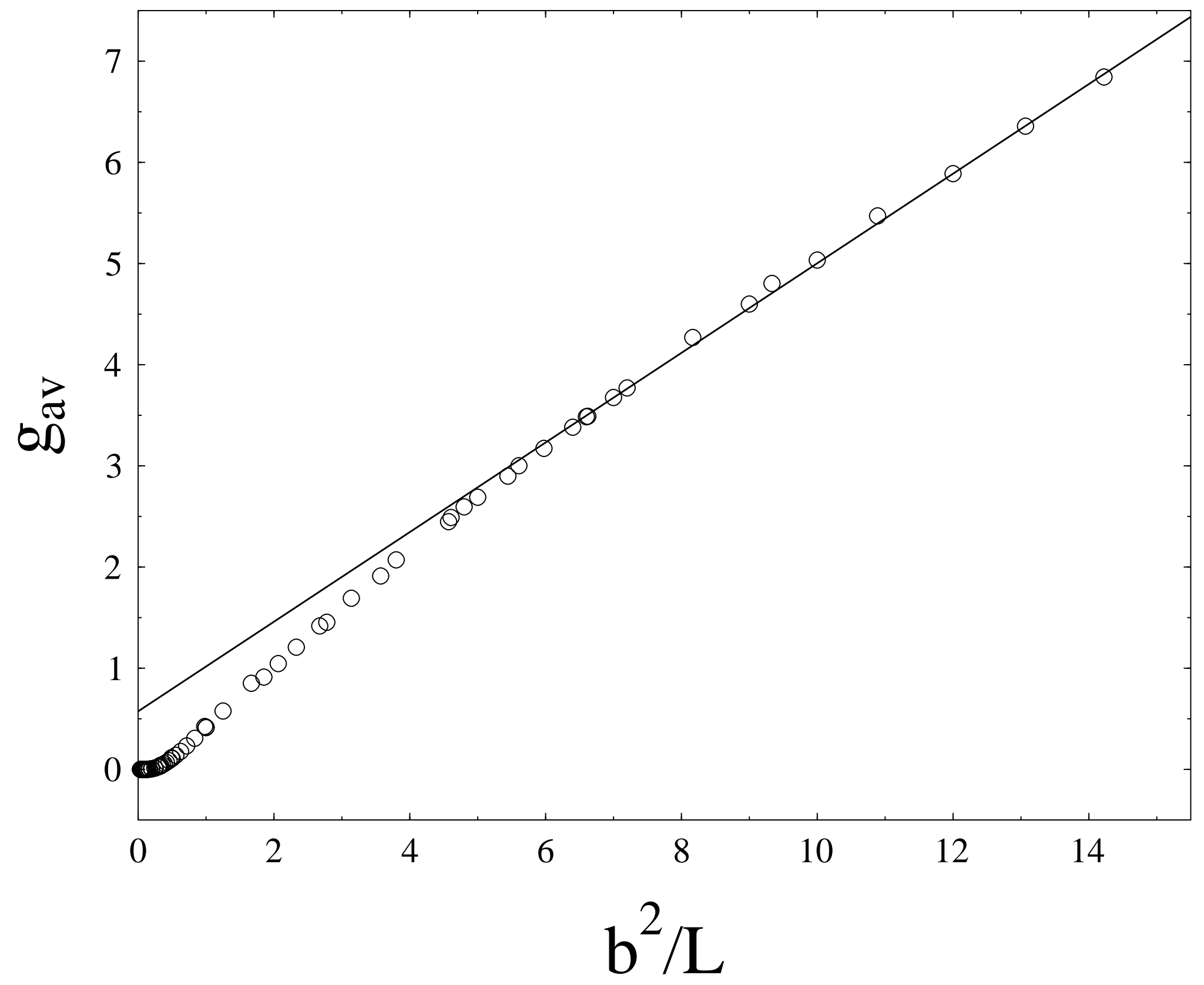




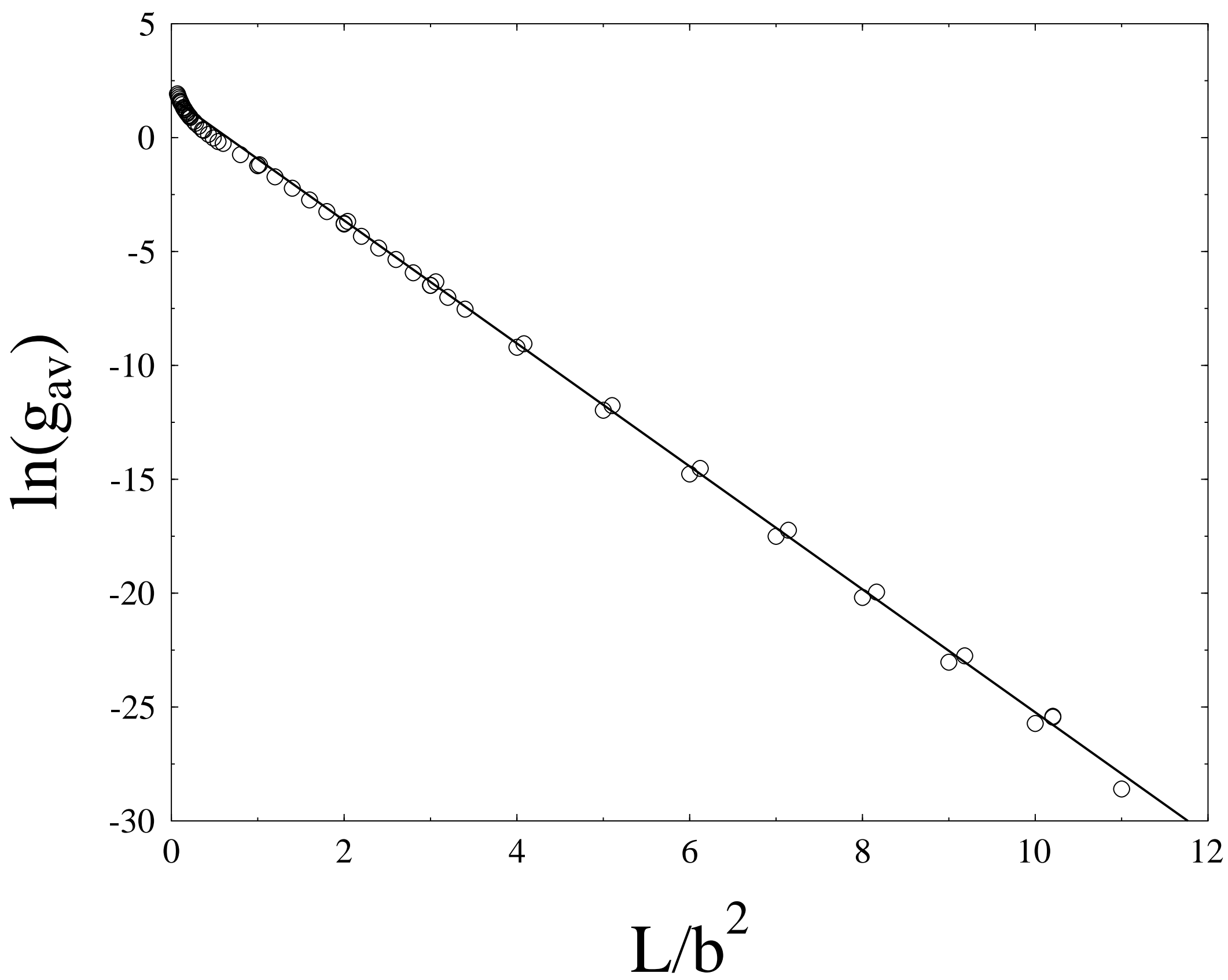




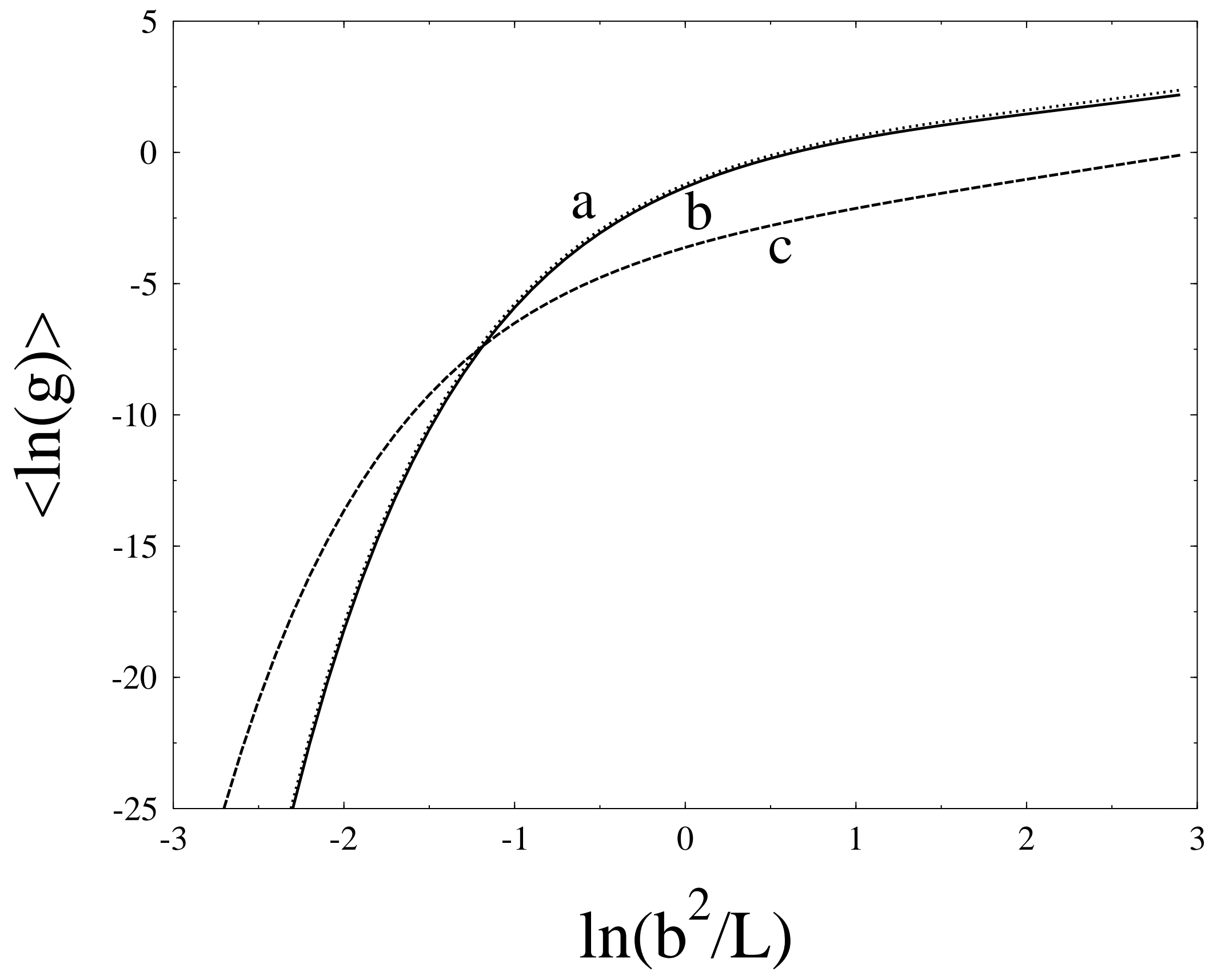




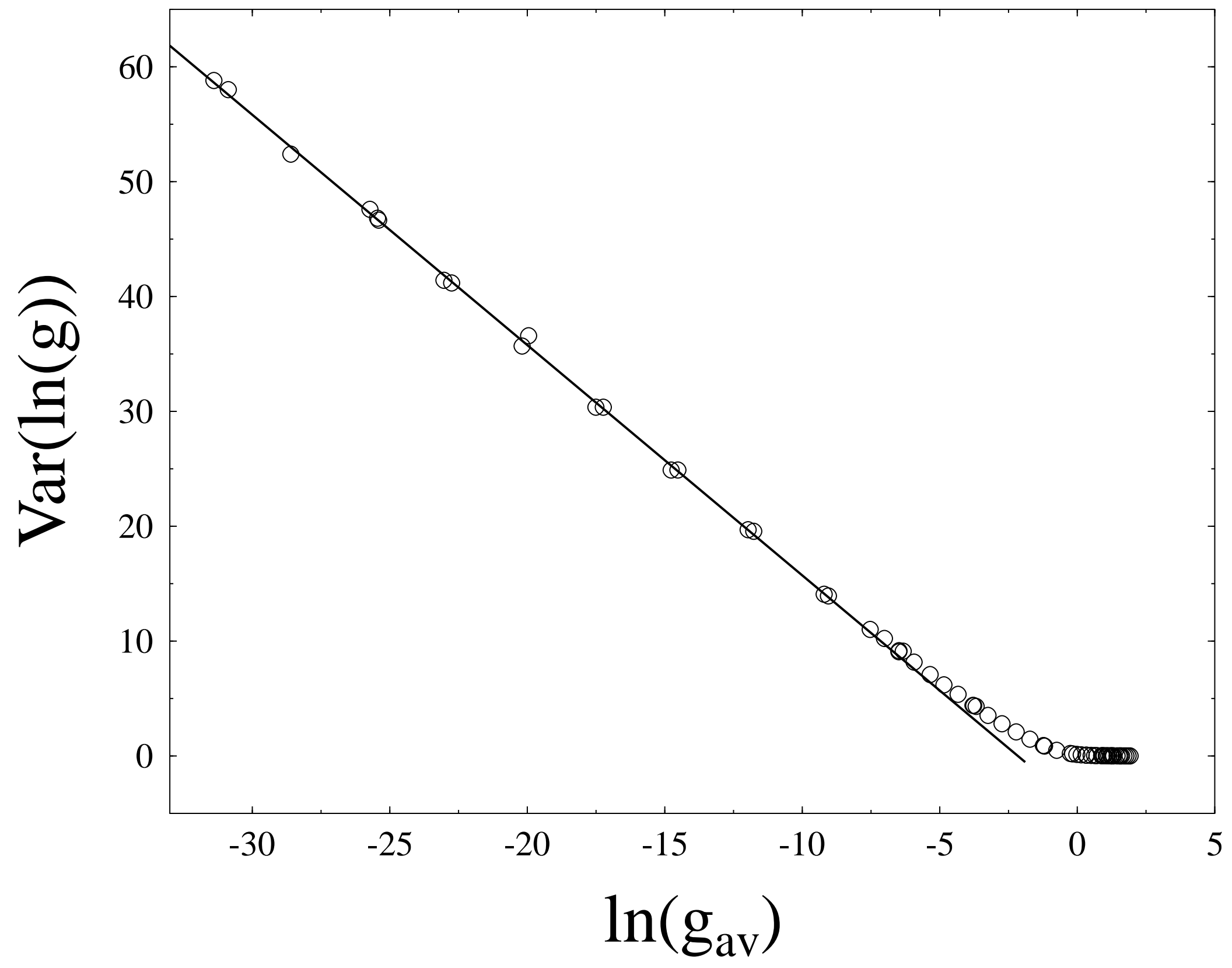




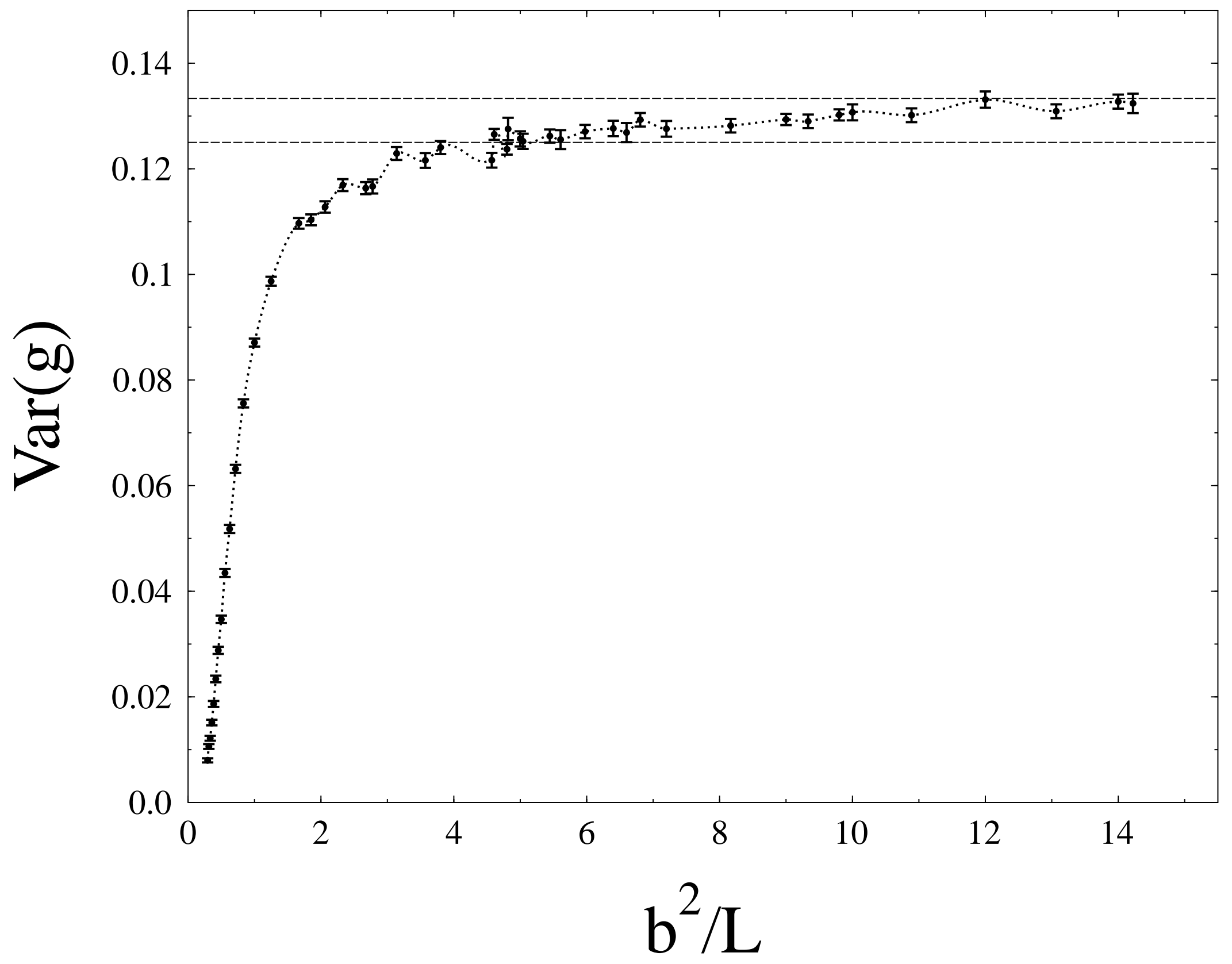

